\title{
Focus point supersymmetry: Proton decay, flavor and $C P$ violation, and the Higgs boson mass
}

\author{
Jonathan L. Feng* \\ Center for Theoretical Physics, Massachusetts Institute of Technology, Cambridge, Massachusetts 02139
}

Konstantin T. Matchev ${ }^{\dagger}$

Theory Division, CERN, CH-1211 Geneva 23, Switzerland

(Received 5 December 2000; published 2 April 2001)

In focus point supersymmetry, all squarks and sleptons, including those of the third generation, have multi-TeV masses without sacrificing naturalness. We examine the implications of this framework for low energy constraints and the light Higgs boson mass. Undesirable contributions to proton decay and electric dipole moments, generic in many supersymmetric models, are strongly suppressed. As a result, the prediction for $\alpha_{s}$ in simple grand unified theories is $3 \sigma-5 \sigma$ closer to the experimental value, and the allowed $C P$-violating phases are larger by one to two orders of magnitude. In addition, the very heavy top and bottom squarks of focus point supersymmetry naturally produce a Higgs boson mass at or above $115 \mathrm{GeV}$ without requiring heavy gauginos. We conclude with an extended discussion of issues related to the definition of naturalness and comment on several other prescriptions given in the literature.

DOI: 10.1103/PhysRevD.63.095003 PACS number(s): 12.60.Jv, 12.10.Kt, 14.20.Dh, 14.80.Bn

\section{INTRODUCTION}

Among the motivations for supersymmetric extensions of the standard model are three important virtues: they provide a natural solution to the gauge hierarchy problem [1-4]; they predict a suitable particle candidate for cold dark matter $[5,6]$; and they incorporate the unification of coupling constants [7]. All three of these virtues are realized in a straightforward way if superpartners masses are of the order of the weak scale. At present, however, no superpartners have been discovered at colliders. Even more problematic, their virtual effects on low energy observables have also not been seen. The incompatibility of generic supersymmetric models with low energy constraints encompasses a diverse set of difficulties, which together are known as the supersymmetric flavor and $C P$ problems.

Much of supersymmetric model building is motivated by the desire to solve these problems without sacrificing some or all of the virtues mentioned above. There are many approaches to this puzzle. Typically, the preservation of naturalness is assumed to require superpartner masses below 1 $\mathrm{TeV}$. The supersymmetric flavor problems are then solved, for example, by scalar degeneracy. Dynamical mechanisms guaranteeing scalar degeneracy have been found. The virtues of these mechanisms are many, but there are also typically a number of attendant difficulties, such as the $\mu$ problem in gauge-mediated models [8] and the problem of tachyonic sleptons in anomaly mediated models [9]. In addition, the suppression of $C P$ violation usually requires additional structure (see, for example, Refs. [9-13]), and the most natural dark matter particle, a neutralino with the desired thermal relic density, is almost always eliminated (although new dark matter candidates may emerge [14-16]).

Focus point supersymmetry $[17-19,13,20]$ has been pro-

\footnotetext{
*Email address: jlf@mit.edu

${ }^{\dagger}$ Email address: konstantin.matchev@cern.ch
}

posed as an alternative to these approaches. In focus point supersymmetry, all squarks and sleptons, including those of the third generation, naturally have masses well above 1 $\mathrm{TeV}$. All supersymmetric flavor and $C P$ problems are then ameliorated by decoupling, while preserving all of the virtues listed above. The naturalness of super-TeV scalars arises from correlations among supersymmetry parameters. More specifically, the weak scale value of the parameter $m_{H_{u}}^{2}$, and with it, the scale of electroweak symmetry breaking, is highly insensitive to the values of the scalar masses $m_{i}$, and is determined primarily by gaugino masses $M_{i}$ and trilinear scalar couplings $A_{i}$. Assuming a hierarchy $m_{i} \gg M_{i}, A_{i}$, as follows naturally from, for example, an approximate $R$ symmetry [21], the observed weak scale may then be obtained without large fine tuning, even in the presence of very large scalar masses.

The conditions for the realization of focus point supersymmetry imply testable correlations in the superpartner mass spectrum. Sufficient conditions have been presented in Ref. [17]. For example, for any value of $\tan \beta \geq 5$ and $m_{t}$ $\approx 174 \mathrm{GeV}$, a universal scalar mass guarantees focus point supersymmetry. ${ }^{1}$ The simplicity of the required scalar mass boundary condition, and the strong dependence of this simplicity on concrete experimental facts, in particular, the measured top quark mass, provide two of the more striking motivations for the framework.

In this respect, the motivation for focus point supersym-

\footnotetext{
${ }^{1}$ In fact, focus point supersymmetry relies on only a small subset of the universality assumption, being independent of all scalar masses with small Yukawa couplings $[17,18]$. In addition, no relations are required among the gaugino masses and $A$ parameters, and supersymmetry breaking need not be gravity mediated. For these reasons, focus point supersymmetry encompasses a broad class of models, and may be found in models with gauge and anomaly mediated supersymmetry breaking $[13,20]$.
} 
metry shares many features with a well-known precedentthe argument for supersymmetric grand unified theories (GUT's). Recall that, assuming minimal supersymmetric field content, the renormalization group (RG) trajectories [22] of the three standard model gauge couplings focus to a point at the scale $M_{\mathrm{GUT}} \simeq 2 \times 10^{16} \mathrm{GeV}$ [23]. This intersection is highly nontrivial. Assuming supersymmetric thresholds around the $\mathrm{TeV}$ scale, there are no free parameters, and the meeting requires the standard model gauge couplings to be within a few percent of their precisely measured values. This may be regarded as a coincidence. However, it may also be taken as evidence for supersymmetry with grand unification, especially as grand unification provides a simple and elegant explanation of the standard model gauge structure and representation content [24]. Indeed, this precise quantitative success has been taken by some as an important advantage of supersymmetry over all other attempts to address the gauge hierarchy problem.

Focus point supersymmetry is motivated by a similar argument. Assuming a universal GUT scale scalar mass, the family of $m_{H_{u}}^{2} \mathrm{RG}$ trajectories for different values of this universal mass meet at a point, the weak scale. This meeting is also highly nontrivial. Assuming unification at the GUT scale, there are no free parameters, and the meeting requires the precisely measured top quark mass to be within $\sim 2 \%$ $(\sim 1 \sigma)$ of its measured value [18]. This may be regarded as a coincidence. However, it may also be taken as evidence for supersymmetry with a large universal scalar mass, especially if it provides a simple solution to the longstanding supersymmetric flavor and $C P$ problems. ${ }^{2}$

In two studies with Wilczek [25,26], we explored the cosmological and astrophysical implications of focus point supersymmetry. In particular, we found that the focus point framework preserves the most natural supersymmetric dark matter candidate, the stable neutralino with the desired thermal relic density. However, unlike traditional scenarios in which this neutralino is $B$-ino-like, in focus point models it is a gaugino-Higgsino mixture. The Higgsino component has important implications for dark matter searches. For example, many indirect detection signal rates are enhanced by several orders of magnitude. The focus point scenario therefore predicts observable signals in diverse experiments, ranging from neutrino and gamma-ray telescopes to space-based searches for antiparticles in cosmic rays.

In this study, we address the following question: to what extent can all of the supersymmetric flavor and $C P$ problems be solved in focus point supersymmetry by heavy scalars? We consider the example of a universal scalar mass in minimal supergravity. In this simple realization of focus point supersymmetry, many supersymmetric flavor problems are solved by assumption. However, even theories with universal

\footnotetext{
${ }^{2}$ This analogy highlights the similarity of focus point supersymmetry and gauge coupling unification in their strong dependence on precisely measured experimental data. Note, however, that in the case of focus point supersymmetry, the meeting is of a family of $\mathrm{RG}$ trajectories, of which only one can be realized in nature.
}

scalar masses generically violate current bounds on proton decay and electric dipole moments, and they may also be significantly constrained by measurements of the muon's magnetic dipole moment and $B \rightarrow X_{s} \gamma$. We will evaluate the status of focus point supersymmetry with respect to each of these constraints in Secs. II-V.

Of course, focus point supersymmetry also has important implications for high energy colliders. The prospects for discovering multi-TeV squarks at the LHC have been considered in Refs. [27] and [28]. In Sec. VI we consider the implications of focus point supersymmetry for discovery of the light Higgs boson. Of all of the as-yet-undiscovered particles of the minimal supersymmetric model, the Higgs boson is of special interest, given current stringent constraints on its mass, the recently reported evidence for its observation at the CERN $e^{+} e^{-}$collider LEP, and the prospect for discovery at Fermilab Tevatron run II. Focus point supersymmetry differs from all other proposed supersymmetric models in that all squarks and sleptons, including the top and bottom squarks, may be naturally heavy. For this reason, focus point supersymmetry has novel implications for the Higgs boson mass. We will show that large radiative corrections from super$\mathrm{TeV}$ squarks naturally lead to Higgs boson masses in the experimentally preferred range.

Finally, we close with an extended discussion of naturalness in Sec. VII. While no discussion of naturalness and fine tuning can claim quantitative rigor, the possibility of focus point supersymmetry raises a number of qualitatively novel issues. When confronted with these issues, various naturalness prescriptions in the literature yield qualitatively different results that should not be dismissed as merely subjective ambiguities. In this section, we compare our approach with others currently in the literature to clarify and highlight the essential differences. We also reiterate that the focus point is valid for all values of $\tan \beta \geq 5$. In Ref. [18], we demonstrated this analytically for moderate $\tan \beta$ and $\tan \beta$ $\approx m_{t} / m_{b}$ and numerically for all $\tan \beta$. In the Appendix we supply the analytical proof for all $\tan \beta \gtrsim 5$.

\section{PROTON DECAY AND GAUGE COUPLING UNIFICATION}

Constraints from proton decay and the status of gauge coupling unification are intimately connected. As reviewed above, the apparent unification of gauge couplings in supersymmetry has long been considered an important virtue. The advantage of the minimal supersymmetric standard model over the standard model with respect to gauge coupling unification is twofold. First, the gauge couplings unify more accurately. This simplifies attempts to build GUT models, since abnormally large threshold corrections are not required. Second, the unification scale $M_{\mathrm{GUT}}$ is high enough that proton decay, mediated by GUT scale particles [29], is sufficiently suppressed to evade experimental bounds.

The current status of supersymmetric unification is, however, significantly more complicated. Analyses of gauge coupling unification now include two-loop RG equations [30] and leading-log [31] and finite $[32,33]$ weak scale threshold corrections. In addition, the measurement of $\sin ^{2} \theta_{W}$ has im- 


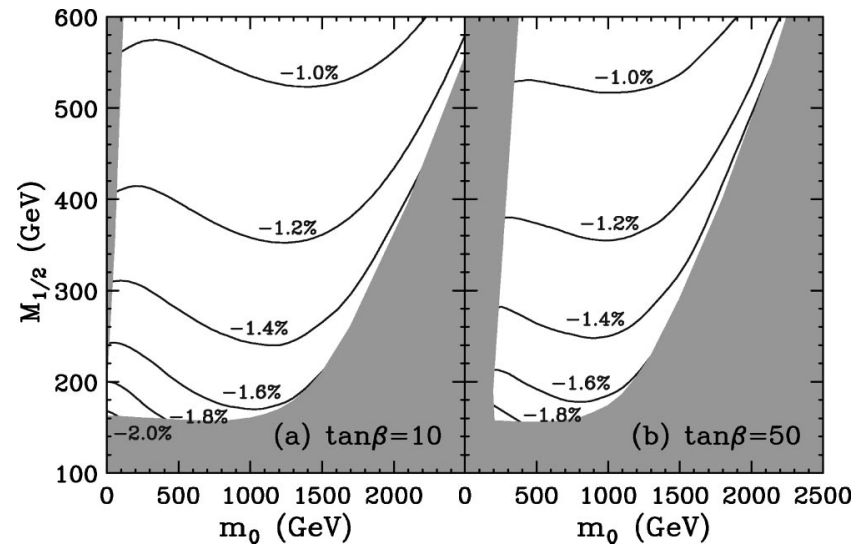

FIG. 1. Contours of $\varepsilon$, the GUT scale mismatch in gauge coupling unification. The shaded regions are excluded by the requirements of a neutral lightest supersymmetric particle (LSP) (left) and the $103 \mathrm{GeV}$ chargino mass bound (right and bottom). In this and all following plots unless otherwise noted, we fix $m_{t}=174 \mathrm{GeV}$, $A_{0}=0$ and $\mu>0$, and choose representative values of $\tan \beta$ as indicated.

proved. With these refinements, the gauge couplings are found to miss each other with a significant discrepancy. Defining, as usual, the GUT scale through the relation $g_{1}\left(M_{\mathrm{GUT}}\right) \equiv g_{2}\left(M_{\mathrm{GUT}}\right)$, a quantitative measure of the mismatch is

$$
\varepsilon \equiv \frac{g_{3}\left(M_{\mathrm{GUT}}\right)-g_{1}\left(M_{\mathrm{GUT}}\right)}{g_{1}\left(M_{\mathrm{GUT}}\right)} .
$$

The parameter $\varepsilon$ depends only on measured standard model quantities and the weak scale supersymmetric particle spectrum.

In minimal supergravity, the weak scale spectrum is fixed by $4+1$ parameters: $m_{0}, M_{1 / 2}, A_{0}, \tan \beta$, and $\operatorname{sgn}(\mu)$. We determine the weak scale theory by two-loop RG evolution with full one-loop threshold corrections. The magnitude of $\mu$ is determined by (full one-loop) radiative electroweak symmetry breaking. Values of $\varepsilon$ in this framework are presented in Fig. 1. Heavy superpartners reduce $|\varepsilon|$ [31-33]. One might therefore naively expect $|\varepsilon|$ to be minimized in the focus point region with large $m_{0}$. For fixed $M_{1 / 2},|\varepsilon|$ indeed decreases as $m_{0}$ increases up to about $1 \mathrm{TeV}$. Above $1 \mathrm{TeV}$, however, $|\mu|$ eventually drops, and threshold corrections from light Higgsinos cause $|\varepsilon|$ to increase again. As a result, throughout parameter space, $-2 \%<\varepsilon<-1 \%$ [32-34]. This GUT scale discrepancy is related to $\alpha_{s}\left(M_{Z}\right)$ by the approximate relation

$$
\delta \alpha_{s} \approx 2 \frac{\alpha_{s}^{2}}{\alpha_{G}} \varepsilon \approx 0.7 \varepsilon
$$

The current value of the strong coupling constant is $\alpha_{s}\left(M_{Z}\right)=0.119 \pm 0.002$ [35]. In terms of the experimental uncertainty, then, the mismatch is a $3.5 \sigma$ to $7 \sigma$ effect.

Of course, one might hope that the mismatch in couplings is a reflection of GUT scale threshold corrections. In the simple case of minimal SU(5) [36], for example, the com- bined threshold correction due to the colored Higgs bosons $\mathrm{H}_{3}$, GUT scale gauge bosons, and the Higgs bosons in the $\mathbf{2 4}$ representation is [37-39]

$$
\varepsilon_{H_{3}}=0.3 \frac{\alpha_{G}}{\pi} \ln \left(\frac{M_{H_{3}}}{M_{\mathrm{GUT}}}\right) \text {. }
$$

We see that light colored Higgs bosons may explain the mismatch. However, recent progress in proton decay, both experimental and theoretical, places stringent lower limits on GUT scale particle masses. Recent results from Superkamiokande significantly strengthen limits on the proton lifetime. In the $p \rightarrow K^{+} \bar{\nu}$ channel, for example, the current limit is $\tau\left(p \rightarrow K^{+} \bar{\nu}\right)>1.9 \times 10^{33} \mathrm{yr}[40]$. On the theoretical side, it is now known that there are dimension 5 supersymmetric contributions to proton decay involving right-handed scalars (the so-called $R R R R$ operators) with amplitudes that scale as $\tan ^{2} \beta$ [41-44]. The combined effect of these developments is that the colored Higgs mass $M_{H_{3}}$ is typically required to be far above $M_{\mathrm{GUT}}$, especially for large $\tan \beta$, and the mismatch in gauge couplings is, in fact, exacerbated by such GUT scale threshold corrections.

In nonminimal models there will be additional GUT threshold corrections. These corrections may improve the unification of couplings [45-47], make it even more problematic $[48,49]$, or be sufficiently complicated that no definite statement can be made [50]. In general, we may write the total GUT threshold correction as $[46,47]$

$$
\varepsilon_{H_{\mathrm{eff}}}+\Delta \varepsilon \equiv 0.3 \frac{\alpha_{G}}{\pi} \ln \left(\frac{M_{H_{\mathrm{eff}}}}{M_{\mathrm{GUT}}}\right)+\Delta \varepsilon
$$

where $M_{H_{\text {eff }}}$ is the effective color triplet Higgs mass entering the proton decay amplitude, and $\Delta \varepsilon$ is the threshold correction from sectors of the theory that have no impact on proton decay. $\Delta \varepsilon$ is generically a model-dependent holomorphic function of ratios of GUT scale masses and vacuum expectation values. For some models, however, $\Delta \varepsilon$ simplifies tremendously. For example, in missing partner SU(5) models [45], $\Delta \varepsilon=0.3\left[\alpha_{G} / \pi\right][15 \ln 2-(25 / 2) \ln 5] \sim-3.9 \%[38,39]$, and in the complete $\mathrm{SO}(10)$ model of Ref. [48], $\Delta \varepsilon$ $=0.3\left[\alpha_{G} / \pi\right] 21 \ln 2 \sim+5.8 \%$ [46]. In both cases, there are no remaining free parameters.

These examples illustrate that the severity of the proton decay problem is model dependent; some specific models may even be consistent with current constraints. However, it is clear, as has recently been emphasized in Ref. [51], that generally speaking, current proton decay bounds place a significant strain on many well-motivated models, as they exclude the large threshold corrections necessary for gauge coupling unification. General mechanisms for suppressing proton decay are therefore welcome, in that they allow greater freedom in GUT model building.

In this spirit, we now investigate the implications of focus point supersymmetry. In Fig. 2, we plot

$$
\Delta \varepsilon \equiv \varepsilon-\varepsilon_{H_{\text {eff }}},
$$




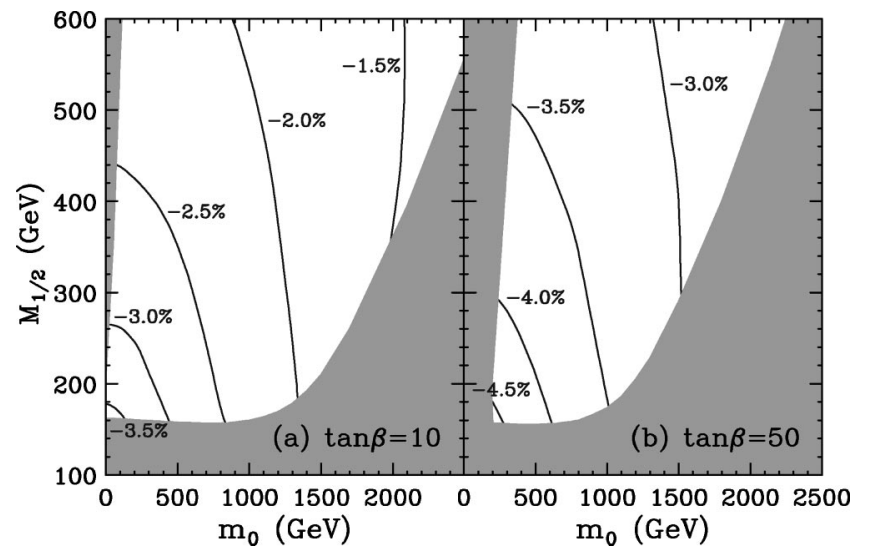

FIG. 2. Contours of the minimal (in absolute value) threshold correction $\Delta \varepsilon$ from nonminimal GUT particle content allowed by coupling constant unification and current proton decay limits.

where $\varepsilon$ is defined in Eq. (1), using weak scale experimental inputs and sparticle spectra, and $M_{H_{\text {eff }}}$ is taken to be as low as possible consistent with current proton lifetime bounds. ${ }^{3}$ In other words, Fig. 2 shows the minimal (in absolute value) threshold correction from non-minimal GUT particle sectors allowed by coupling constant unification and current proton decay constraints.

We see that in the focus point region with large $m_{0}$, coupling constant unification may be achieved with smaller nonminimal threshold corrections. In this region, proton decay is highly suppressed by heavy squarks and sleptons. The allowed value of $M_{H_{\text {eff }}}$ is therefore lower than in conventional models, and the required additional GUT threshold correction from nonminimal GUT sectors is reduced. More quantitatively, for a fixed $M_{1 / 2}$, the required threshold corrections are decreased by $1 \%$ to $1.5 \%$ for focus point scenarios with multi-TeV scalars relative to conventional scenarios with $m_{0} \sim \mathcal{O}(100 \mathrm{GeV})$. Thus in many GUT's, the prediction for $\alpha_{s}\left(M_{Z}\right)$ is closer to the experimental value by $3 \sigma$ to $5 \sigma$ in focus point models relative to conventional scenarios. As a result, in focus point scenarios, large threshold corrections from baroque nonminimal sectors are not required, increasing the viability of simpler and, presumably, more credible models.

\section{ELECTRIC DIPOLE MOMENTS}

Constraints on $C P$ violation can be flavor violating, as in the case of $\epsilon_{K}$, or flavor conserving, as in the case of electric dipole moments (EDM's). For generic theories, the bound from $\epsilon_{K}$ is the most stringent of all flavor- and $C P$-violating constraints. However, the $\epsilon_{K}$ constraint is satisfied in many theories with natural flavor violation suppression. In contrast, the EDM constraints are more robust, in the sense that they cannot be avoided simply by scalar degeneracy or alignment. For this reason, EDM's pose a serious problem even in mod-

\footnotetext{
${ }^{3}$ In evaluating the proton lifetime bound, we use the fits of Ref. [44], which include the $R R R R$ contributions.
}

els with a universal scalar mass, as well as in gauge and anomaly mediated theories. EDM's have been studied in many supersymmetric models. (See, for example, Ref. [12] and references therein.) Here we evaluate the predictions for EDM's in focus point supersymmetry.

In minimal supergravity, the parameters $M_{1 / 2}, A_{0}, \mu$, and $B$ may all be complex. The first two are input parameters of the framework. The $\mu$ and $B$ parameters are constrained by electroweak symmetry breaking, but this restricts only their magnitudes. In principle, it is possible that the phases of these four parameters are related, but lacking any specific mechanism for their generation, we treat them as independent. The freedom of $\mathrm{U}(1)_{R}$ and $\mathrm{U}(1)_{P Q}$ rotations imply that only two phases are physical. One of these is

$$
\theta_{\mathrm{CP}} \equiv \operatorname{Arg}\left(\mu B^{*} M_{1 / 2}\right),
$$

which generates EDM's. The EDM $d_{f}$ of fermion $f$ is the coefficient of the electric dipole term

$$
\mathcal{L}_{\mathrm{EDM}}=-\frac{i}{2} d_{f} \bar{f} \sigma^{\alpha \beta} \gamma_{5} f F_{\alpha \beta},
$$

where $F$ is the electromagnetic field strength. Supersymmetric contributions to EDM's arise from sfermion-gaugino loops. As is clear from the structure of the operator in Eq. (7), these contributions require a chirality flip along the fermion-sfermion line. For down-type fermions, these contributions are therefore enhanced for large $\tan \beta$.

We will consider the stringent constraints from the EDM's of the electron and neutron. (The EDM of the mercury atom is also competitive in some regions of parameter space [52].) For the electron, there is a direct $\tan \beta$ enhancement. This is most easily seen in the mass insertion approximation, where, for large $\tan \beta$, the supersymmetric contributions take the form

$$
\begin{aligned}
d_{e}^{\mathrm{SUSY}} \approx & \sin \theta_{\mathrm{CP}} \frac{m_{e}}{2} \mu \tan \beta\left[g_{1}^{2} M_{1} F_{1}\left(M_{1}^{2}, \mu^{2}, m_{\tilde{e}_{L}}^{2}, m_{\tilde{e}_{R}}^{2}\right)\right. \\
& \left.+g_{2}^{2} M_{2} F_{2}\left(M_{2}^{2}, \mu^{2}, m_{\tilde{e}_{L}}^{2}, m_{\tilde{\nu}_{e}}^{2}\right)\right],
\end{aligned}
$$

where explicit formulas for the $F$ functions are given in Ref. [53]. For large sfermion masses, $F \sim m_{\tilde{f}}^{-4}$. To calculate the neutron EDM, we must model the structure of the neutron. We adopt the nonrelativistic quark model, in which the neutron EDM is $d_{n}=\left(4 d_{d}-d_{u}\right) / 3$. Contributions to the quark EDM's are similar to those for the electron, with the exception that there are additional contributions from squarkgluino diagrams. Note that, since $d_{d} \propto \tan \beta$, the neutron EDM is also enhanced for large $\tan \beta$.

The standard model predicts vanishing EDM's, to foreseeable experimental accuracy. At present, no anomaly is seen in EDM measurements. From the measurement $d_{e}$ $=(0.18 \pm 0.12 \pm 0.10) \times 10^{-26} e \mathrm{~cm}[54]$, we obtain the constraint $\left|d_{e}\right| \leqslant 0.44 \times 10^{-26} e \mathrm{~cm}$, where the right-hand side is the upper bound on $\left|d_{e}\right|$ at $90 \%$ C.L. For the neutron, the current 90\% C.L. limit is $\left|d_{n}\right| \leqslant 0.63 \times 10^{-25} e \mathrm{~cm}$ [55]. 


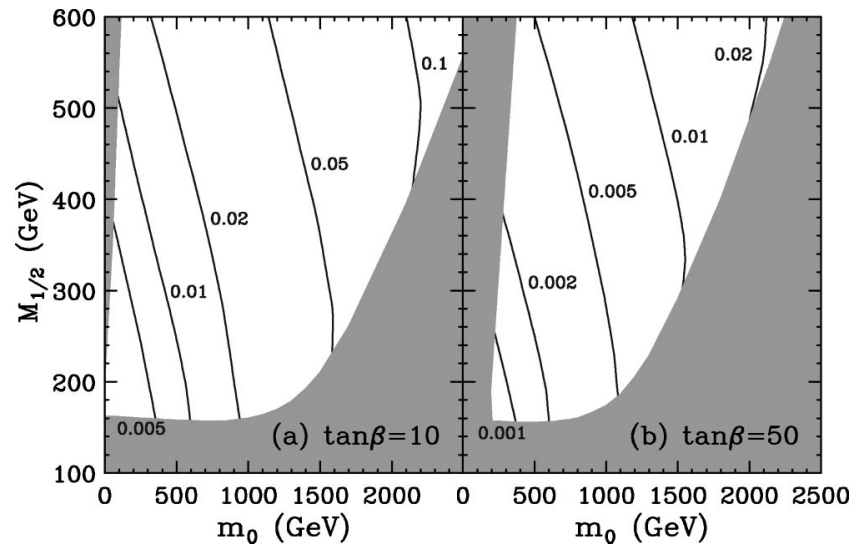

FIG. 3. Contours of the maximum value of $\sin \theta_{\mathrm{CP}}$ allowed by the electron EDM constraint $\left|d_{e}\right| \leqslant 0.44 \times 10^{-26} e \mathrm{~cm}$.

The present constraints on EDM's severely restrict the possible values of $\theta_{\mathrm{CP}}$. In Figs. 3 and 4, we plot the maximal allowed values of $\theta_{\mathrm{CP}}$ given the constraints of the electron and neutron EDM's, respectively. The EDM's are calculated in the exact mass eigenstate basis. We see that current constraints from the electron and neutron are roughly comparable. For sub-TeV values of $m_{0}, \theta_{\mathrm{CP}}$ is constrained to be less than of order $10^{-3}$ to $10^{-2}$, depending on $\tan \beta$. In the absence of an understanding of the origin of this phase, this appears to require a strong fine tuning. For the focus point scenario with multi-TeV $m_{0}$, these constraints may be relaxed by over an order of magnitude. In the $\tan \beta=10$ case, $\mathcal{O}(0.1)$ phases are allowed.

It is important to note that there is some sensitivity to the assumed top mass. For larger top quark mass, but still within the experimental bounds, the excluded region from chargino mass limits moves to larger $m_{0}$, and so even larger scalar masses are allowed. In Fig. 5, we show the $C P$-violating phases allowed by the electron EDM, but with an assumed top quark mass of $m_{t}=179 \mathrm{GeV}$, within the $1 \sigma$ experimental bound. As $m_{0}$ now extends to over $3 \mathrm{TeV}$, even larger phases are allowed. A similar improvement is found in the neutron EDM case.

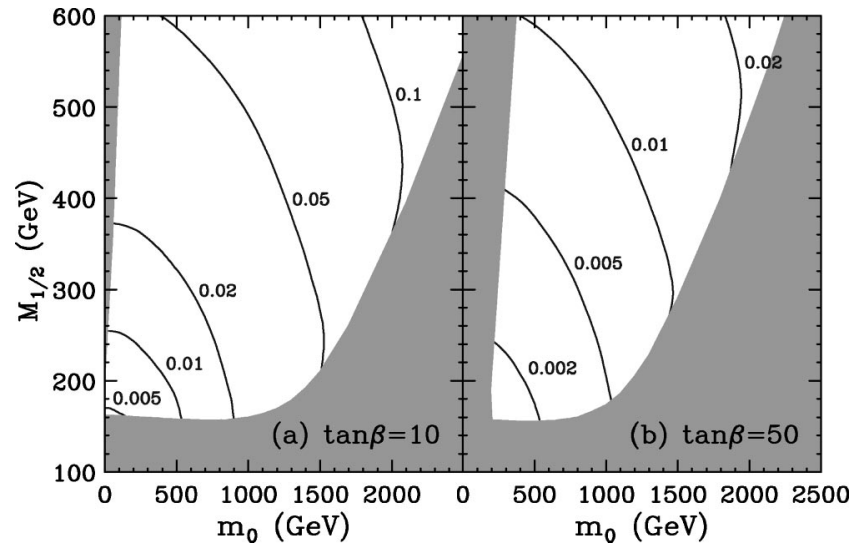

FIG. 4. As in Fig. 3, but for the neutron EDM constraint $\left|d_{n}\right|$ $\leqslant 0.63 \times 10^{-25} e \mathrm{~cm}$.

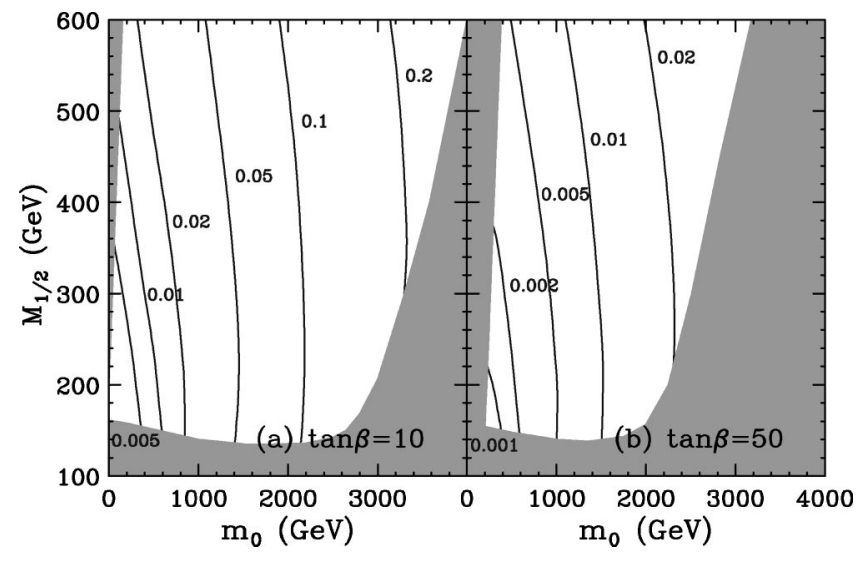

FIG. 5. As in Fig. 3, but for $m_{t}=179 \mathrm{GeV}$.

\section{MAGNETIC DIPOLE MOMENT OF THE MUON}

Supersymmetric particles also contribute radiatively to magnetic dipole moments (MDM's). Such contributions are even more robust than EDM's, as they require neither $C P$ nor flavor violation. At present the most stringent constraint comes from the muon's anomalous MDM $a_{\mu}=\frac{1}{2}(g-2)_{\mu}$, which is the coefficient of the operator

$$
\mathcal{L}_{\mathrm{MDM}}=a_{\mu} \frac{e}{4 m_{\mu}} \bar{\mu} \sigma^{\alpha \beta} \mu F_{\alpha \beta} .
$$

The supersymmetric contributions are similar to those discussed above for the electron EDM, arising from sleptonneutralino and sneutrino-chargino loops. In the large $\tan \beta$ regime, they take the form

$$
\begin{aligned}
a_{\mu}^{\mathrm{SUSY}} \approx & m_{\mu}^{2} \mu \tan \beta\left[g_{1}^{2} M_{1} F_{1}\left(M_{1}^{2}, \mu^{2}, m_{\tilde{\mu}_{L}}^{2}, m_{\tilde{\mu}_{R}}^{2}\right)\right. \\
& \left.+g_{2}^{2} M_{2} F_{2}\left(M_{2}^{2}, \mu^{2}, m_{\tilde{\mu}_{L}}^{2}, m_{\tilde{\nu}_{\mu}}^{2}\right)\right]
\end{aligned}
$$

where in this section, we assume all parameters real. The $F$ functions are as in Eq. (7).

The anomalous MDM of the muon has been measured at CERN [56] and Brookhaven [57,58]. The current world average is $a_{\mu}^{\exp }=\left(\begin{array}{lll}116 & 59205 \pm 45\end{array}\right) \times 10^{-10}$ [58], consistent with the standard model. The uncertainty is statistics dominated, and will be reduced by the ongoing Brookhaven experiment E821. With data already being collected, the uncertainty should be reduced to $\sim 7 \times 10^{-10}$, and the ultimate goal of E821 is $\Delta a_{\mu} \sim 4 \times 10^{-10}$ [59]. The current standard model prediction for the muon's anomalous MDM is $a_{\mu}^{\text {th }}$ $=(11659162 \pm 8) \times 10^{-10}$ [60]. The uncertainty in the prediction is dominated by the difficulty of evaluating the hadronic vacuum polarization contribution, but is being reduced by improved low energy data. If the theoretical prediction is brought under control, a reasonable $2 \sigma$ limit in the near future is $8 \times 10^{-10}$.

The supersymmetric contribution to the muon anomalous MDM $a_{\mu}^{\text {SUSY }}$, in the mass insertion approximation [53], is given in Fig. 6. As expected, the contribution is enhanced for large $\tan \beta$, and highly suppressed by heavy sleptons in the focus point region. A measured deviation is consistent with 


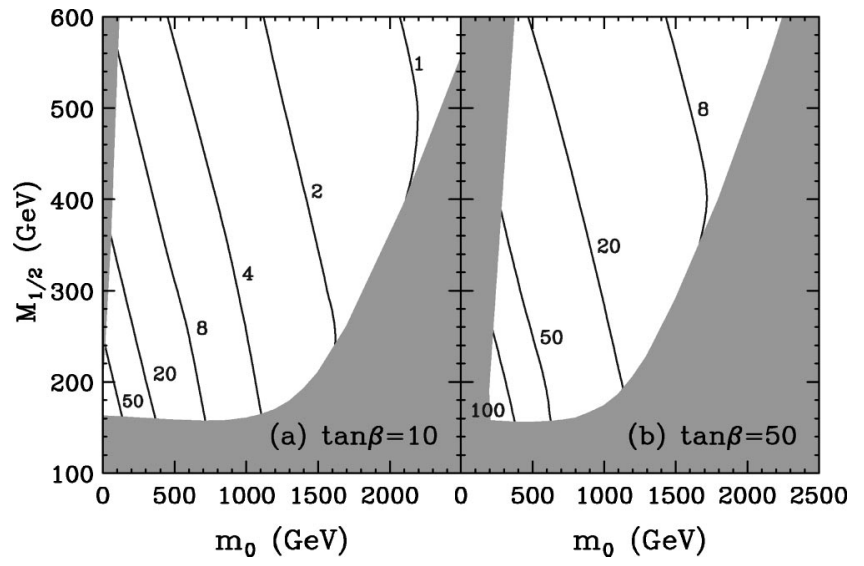

FIG. 6. The muon anomalous MDM $a_{\mu}^{\mathrm{SUSY}}$ in units of $10^{-10}$.

focus point supersymmetry, but only for large $\tan \beta$. On the other hand, if no deviation is found, the muon's anomalous MDM will be a strong argument for heavy superpartners. (Recall that the muon MDM is flavor and $C P$ conserving, so cannot be eliminated by, for example, scalar degeneracy or small phases.) For moderate $\tan \beta$, considerations of dark matter relic density eliminate the moderate $m_{0}$ possibility (see below), and so a muon MDM consistent with the standard model would require $m_{0}$ above a TeV. For large $\tan \beta$, even such a robust cosmological constraint is unnecessary: for $\tan \beta=50$, the absence of an anomaly would require $m_{0}$ $\gtrsim 1.5 \mathrm{TeV}$, well into the focus point region.

$$
\text { V. } B \rightarrow X_{s} \gamma
$$

It is well known that the supersymmetric contributions to $B \rightarrow X_{s} \gamma$ may be large. In the standard model, this flavorviolating transition takes place only at one loop through a $W$ boson. In supersymmetric theories, there are a variety of additional one-loop contributions [61], most importantly those from charged Higgs- and chargino-mediated processes. These are both enhanced by large $\tan \beta$ in focus point supersymmetry. For the chargino diagrams, this is true for the standard reason of enhanced Yukawa couplings. For the charged Higgs diagram, it holds because large $\tan \beta$ implies small charged Higgs boson masses. At small $\tan \beta, m_{H^{+}}$is of order the scalar superpartner masses, and so is well above $1 \mathrm{TeV}$ in the focus point region. However, for large $\tan \beta$, by the approximate up-down symmetry, both $m_{H_{u}}^{2}$ and $m_{H_{d}}^{2}$ have weak scale focus points, and so $m_{H^{+}}$is typically of the order of $100 \mathrm{GeV}$.

We evaluate $B\left(B \rightarrow X_{s} \gamma\right)$ as follows. As we are primarily interested in the case where there is a hierarchy between the scale of the superpartner masses $M_{\text {SUSY }}$ and the weak scale $M_{\text {Weak }}$, we are careful not to decouple the supersymmetric contributions at the weak scale, as is usually done. Instead, we need to resum the large logarithms of $M_{\text {SuSY }} / M_{\text {Weak }}$ [62-64]. Operationally, we evaluate the leading order supersymmetric contributions [61] at the superpartner scale (defined as the geometric mean of the two top squark masses) and evolve them to the weak scale, using leading order anomalous dimension coefficients. At the weak scale, we

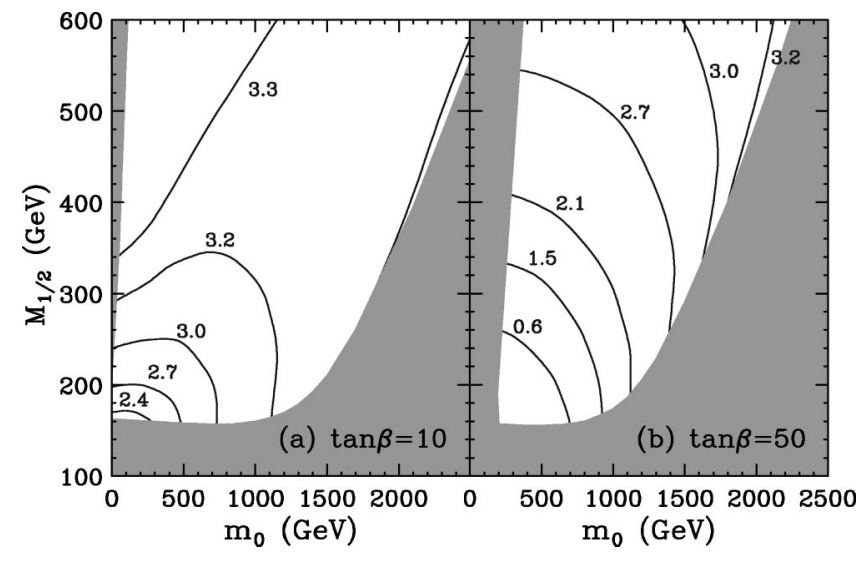

FIG. 7. $B\left(B \rightarrow X_{s} \gamma\right)$ in units of $10^{-4}$ for $\mu>0$.

match these contributions to the effective Hamiltonian

$$
\mathcal{H}_{\mathrm{eff}}=-\frac{4 G_{F}}{\sqrt{2}} V_{t s}^{*} V_{t b} \sum_{i=1}^{8} C_{i} \mathcal{O}_{i}
$$

We use next-to-leading order matching conditions for the standard model [65] and charged Higgs [66] contributions.

The weak scale Wilson parameters $C_{i}$ must then be evolved to the low energy scale $\mu_{b}$ with the NLO anomalous dimension matrix [67], and $B\left(B \rightarrow X_{s} \gamma\right)$ is then evaluated using NLO matrix elements [68], incorporating the leading order QED and electroweak radiative corrections [69,70]. These results have been included in a simple parameterization of Ref. [70], which we adopt, taking $\mu_{b}=m_{b}$ and a photon energy cutoff parameter $\delta=0.9$.

The best current measurements of $B \rightarrow X_{s} \gamma$ from CLEO [71] and ALEPH [72] may be combined in a weighted average of $B\left(B \rightarrow X_{s} \gamma\right)_{\exp }=(3.14 \pm 0.48) \times 10^{-4}[70]$. It is expected that these measurements will be significantly improved at the $B$ factories, where large samples of $B$ mesons will greatly reduce statistical errors. At present, the standard model prediction is $B\left(B \rightarrow X_{s} \gamma\right)_{\mathrm{SM}}=(3.29 \pm 0.30) \times 10^{-4}$ [70]. The theoretical uncertainty is less likely to improve substantially. We estimate that in the near future, both theoretical and experimental uncertainties will be $\sim 0.3 \times 10^{-4}$. Combining these errors linearly, the resulting $2 \sigma$ limit will be $2.1 \times 10^{-4}<B\left(B \rightarrow X_{\text {s }} \gamma\right)<4.5 \times 10^{-4}$.

In Figs. 7 and 8 , we plot contours of $B\left(B \rightarrow X_{s} \gamma\right)$ for positive and negative $\mu$, respectively. ${ }^{4}$ The charged Higgs contribution is always constructive with the standard model. For $\mu<0$, the chargino contribution is also constructive, and predicted $B \rightarrow X_{s} \gamma$ rates are enhanced. For $\mu>0$, the chargino contribution flips sign, and may cancel the charged Higgs contribution.

\footnotetext{
${ }^{4}$ We do not show results for $\tan \beta=50$ and $\mu>0$. For such parameters, difficulties in obtaining correct electroweak symmetry breaking exclude much of the parameter space, and for the remaining region, the prediction for $B \rightarrow X_{s} \gamma$ is always very large and excluded by current bounds.
} 


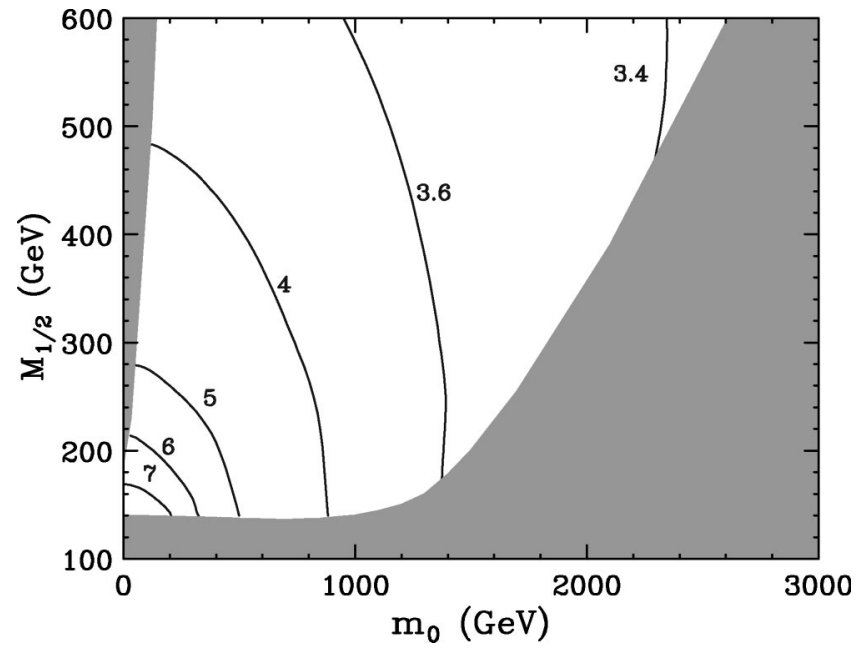

FIG. 8. As in Fig. 7, but for $\mu<0$ and $\tan \beta=10$.

We see that for the foreseeable future, focus point supersymmetry predicts no measurable deviation from the standard model, and both positive $\mu$ and negative $\mu$ (with moderate $\tan \beta$ ) are consistent if no deviation is seen. Of course, if no deviation is found, supersymmetric models with sub$\mathrm{TeV}$ scalar mass $m_{0}$ are also consistent for moderate and low $\tan \beta$.

\section{THE MASS OF THE LIGHT HIGGS BOSON}

The implications of focus point supersymmetry for the light Higgs boson are of special interest, given the present bound of $m_{h}>113.5 \mathrm{GeV}$, and the recent observation at LEP of a $2.9 \sigma$ excess of events consistent with the production of a standard model-like Higgs boson with mass $m_{h}=115 \mathrm{GeV}$ $[73,74]$.

As is well known, in the minimal supersymmetric model, the light Higgs boson mass satisfies $m_{h} \lesssim 130 \mathrm{GeV}$. This limit is saturated in regions of parameter space, where, for example, trilinear $A$ parameters are adjusted to give maximal left-right scalar mixing in the third generation squarks. Such regions are, however, extraordinarily unnatural, requiring extreme fine tuning in the electroweak potential [18]. In fact, in natural regions of parameter space with $m_{0} \lesssim 1 \mathrm{TeV}$, Higgs boson masses as high as those presently preferred are already highly constraining. In Ref. [75], the authors concluded that a Higgs boson mass of $115 \mathrm{GeV}$, along with the assumption of a suitable $B$-ino-like dark matter candidate, implied lower limits on gaugino masses, with strong (negative) implications for supersymmetry searches at the Tevatron. In Ref. [76], similar considerations led the authors to consider, among other possibilities, large $C P$ violating phases, which much necessarily cancel to high accuracy in EDM's.

In focus point supersymmetry, all squarks and sleptons, including those of the third generation, may be above $1 \mathrm{TeV}$ without significantly increased fine-tuning in the electroweak potential. This is in contrast to all other proposed models, including those that also make use of RG effects to resolve the tension between low energy constraints and naturalness, but which, while allowing heavy first and second generation

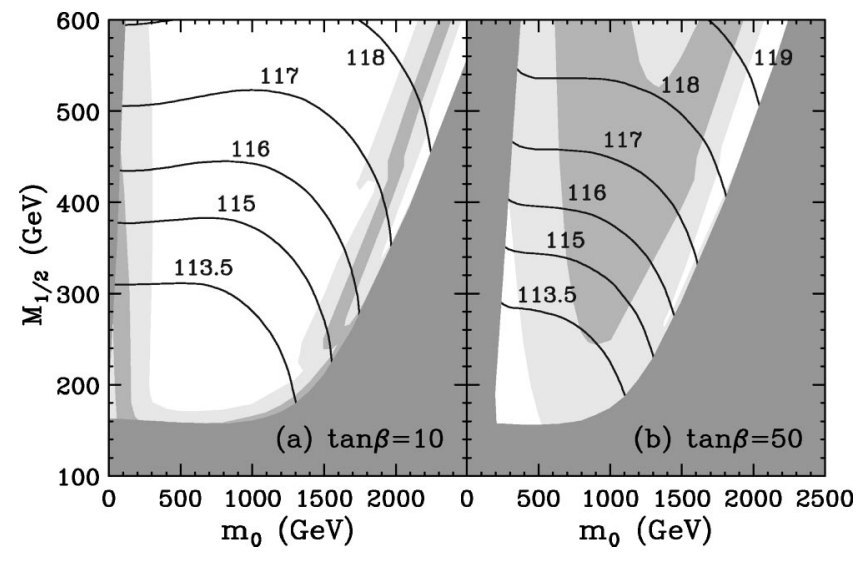

FIG. 9. Contours of Higgs mass $m_{h}$ in GeV. Regions with allowed and preferred dark matter relic density are also shown. In the light shaded region, the thermal relic density of the neutralino LSP is $0.025 \leq \Omega_{\chi} h^{2} \lesssim 1$, and in the dark shaded region it is in the preferred range $0.1 \leqq \Omega_{\chi} h^{2} \leqq 0.3$. The unshaded region above the preferred band has $\Omega_{\chi} h^{2} \gtrsim 1$ and is excluded.

scalars, require light third generation superpartners [77]. As the dominant radiative contributions to the light Higgs boson mass are logarithmically dependent on top and bottom squark masses, this fact has strong implications for the Higgs boson. In Fig. 9, we present contours of constant Higgs boson mass, including the full one-loop radiative corrections as in Ref. [34]. We see that Higgs boson masses at or above $115 \mathrm{GeV}$ are naturally and simply accommodated in the focus point region. In fact, a Higgs boson with mass consistent with present bounds is an inescapable consequence of focus point supersymmetry with multi-TeV squarks. Varying $A_{0}$ within a generous range allowed by naturalness does not change these conclusions [18]. In Fig. 10 we illustrate the dependence on the top quark mass. Variations of $m_{t}$ within its $1 \sigma$ experimental uncertainty give rise to $\sim 2 \mathrm{GeV}$ variations in $m_{h}$.

Note that the focus point region possesses a suitable neutralino dark matter candidate, a Higgsino-gaugino mixture. In Fig. 9, we show also the regions with good thermal relic density. In conventional scenarios, with $m_{0} \lesssim 1 \mathrm{TeV}$ as-

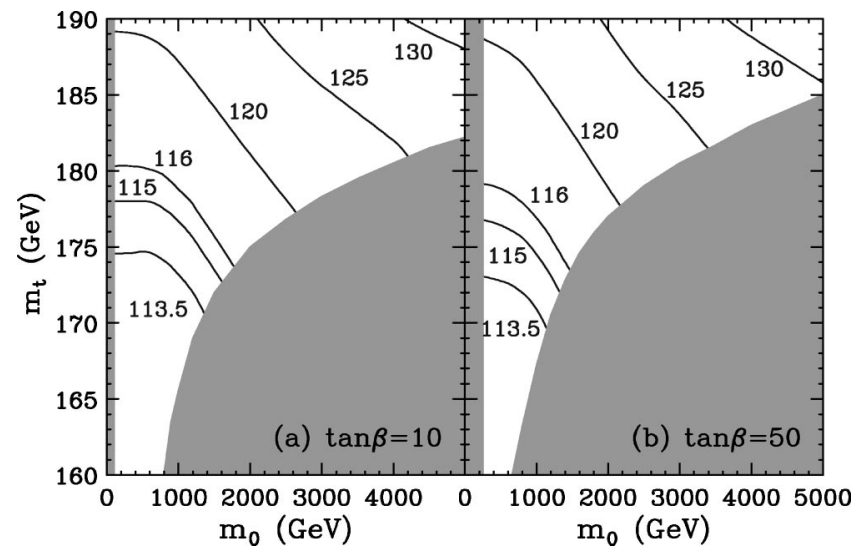

FIG. 10. Contours of Higgs boson mass $m_{h}$ in $\mathrm{GeV}$ in the $\left(m_{0}, m_{t}\right)$ plane for fixed $M_{1 / 2}=300 \mathrm{GeV}, A_{0}=0$, and $\mu>0$. 
sumed, the LSP is $B$-ino-like, and the thermal relic density constrains $m_{0}$ to values of at most $\sim 200 \mathrm{GeV}$. The radiative corrections from $m_{0}$ to the Higgs boson mass are therefore small, and present bounds already require large $M_{1 / 2}$. However, as noted in Refs. [25] and [26], the assumption of a $B$-ino-like LSP is far from robust, and is violated even in the simple framework of minimal supergravity. From Fig. 9, we see that a cosmologically attractive region exists in the focus point region, with $m_{0}>1 \mathrm{TeV}$. In this region, the LSP is a gaugino-Higgsino mixture, and its relic density may also be in the preferred range $0.1 \leqq \Omega_{\chi} h^{2} \leq 0.3$. The focus point region therefore provides an excellent dark matter candidate in which the Higgs boson mass is naturally in the currently preferred range.

\section{ON NATURALNESS}

In the preceding sections, we have found several phenomenological virtues of focus point scenarios with respect to proton decay, the supersymmetric flavor and $C P$ problems, and light Higgs boson mass. Such attractive features would be offset by the ugliness of a fine-tuned electroweak scale, were it not for the focus point mechanism, which makes heavy scalars natural. In this section, we attempt to clarify several issues concerning naturalness by comparing our prescription with several others in the literature. Naturalness has been discussed in a large number of studies. In the following, we do not attempt a comprehensive review, but rather highlight various similarities and differences between our prescription and selected other studies [78-85].

All definitions of naturalness are open to quantitative ambiguities. However, this fact should not be allowed to obscure the many strong qualitative differences that, as we will see, exist between various naturalness prescriptions. The claim that the focus point renders multi-TeV scalars natural is qualitatively novel, and leads to qualitatively new implications for many searches for supersymmetry. For this reason, it is worthwhile to identify and explore the underlying differences between our prescription and others in the literature. As a by-product, we also highlight many issues in defining naturalness that are seldom addressed.

\section{A. Our prescription}

We begin by briefly reviewing our naturalness prescription. Readers interested in a more careful and detailed description are referred to Refs. [17,18]. The five step prescription is the following:

(1) Choose a supersymmetric model framework. For example, if one chooses minimal supergravity, one assumes input parameters $\left\{m_{0}, M_{1 / 2}, A_{0}, \tan \beta, \operatorname{sgn}(\mu)\right\}$ and adopts all the assumptions encapsulated in these $4+1$ parameters.

(2) For a given set of input parameters, determine all weak scale parameters of the theory consistent with experimental data and RG evolution.

(3) Choose some set of parameters to be free, continuously variable, independent, and fundamental. In minimal supergravity, we choose the GUT scale parameters $\left\{a_{i}\right\}$ $=\left\{m_{0}, M_{1 / 2}, A_{0}, B_{0}, \mu_{0}\right\}$. Note that we have included all pa- rameters expected to be intimately related to supersymmetry breaking, but none of the others.

(4) For each fundamental parameter, define the sensitivity coefficient ${ }^{5}[78]$

$$
c_{i} \equiv\left|\frac{\partial \ln m_{Z}}{\partial \ln a_{i}}\right|=\left|\frac{a_{i}}{m_{Z}} \frac{\partial m_{Z}}{\partial a_{i}}\right| .
$$

(5) Finally, define the overall measure of fine tuning to be

$$
c=\max \left\{c_{i}\right\} .
$$

\section{B. Sensitivity coefficients}

The sensitivity coefficients of Eq. (12) are the kernel of most naturalness prescriptions. They were first advanced as a tool for quantifying naturalness by Ellis, Enqvist, Nanopoulos, and Zwirner [78]. These authors analyzed an $E_{6}$ model with superpotential $W=h_{t} Q_{3} U_{3}^{c} H+\lambda H \bar{H} N+k D D^{c}$, where the first term is the top quark Yukawa coupling, $N$ is a singlet Higgs field, $H$ and $\bar{H}$ are the standard Higgs doublets, and $D$ and $D^{c}$ are exotic down-type quarks. They then defined sensitivity coefficients $c_{i}=\left|\partial \ln x / \partial \ln a_{i}\right|$, where $x$ $\equiv\langle N\rangle /\langle H\rangle$, and used $c_{\lambda}, c_{k}<5$ as a reasonable requirement for natural regions of parameter space.

Aside from a difference in the framework being examined, our naturalness definition differs from this one only in what parameter has been chosen to represent the weak scale (their parameter $x$ vs our $m_{Z}^{2}$ ). This difference is minimal, and these prescriptions are identical in spirit. Note that the sensitivity to the standard model parameter $h_{t}$ was not included.

\section{Model dependence and the choice of fundamental parameters}

In another pioneering study, the sensitivity coefficients were then used by Barbieri and Giudice to examine naturalness in the context of minimal supergravity [79]. In that paper, an overall fine-tuning parameter similar to that defined in Eq. (13) was used: the sensitivities to all supersymmetrybreaking parameters (and $\mu_{0}$ ) were included, but sensitivities to standard model parameters were not. These authors considered a range of $h_{t}$ and ignored the effects of $h_{b}$. For particular $h_{t}$, the weak scale was found to be insensitive to variations in $m_{0}$, and in fact, they found singularities in figures plotting the naturalness limits on $m_{0}$. These singularities result from the same numerical "coincidence" responsible for the focus point mechanism. ${ }^{6}$ However, although the sensitivity to $h_{t}$ was not included in the numerical analysis, these authors expected a full analysis of naturalness to include this sensitivity [87], and noted that the singularities of

\footnotetext{
${ }^{5}$ This formula corrects a typographical error in the definition of $c_{i}$ in Refs. [17,18].

${ }^{6}$ This numerical fact can also be deduced from earlier papers studying the RG behavior of minimal supergravity. (See, for example, Ref. [86].)
} 
the figures would be eliminated if the sensitivity to $h_{t}$ were included. (The sensitivity of the weak scale to the top Yukawa coupling was considered in more detail in later studies—see, for example, Ref. [83].) For this reason, these authors did not claim that multi-TeV scalars could be natural. Of course, at that time, the top quark mass was only indirectly bounded, and for most possible masses, the inclusion of $c_{h_{t}}$ made no qualitative difference to the results.

After the discovery of the top quark and the measurement of its mass, studies of naturalness and the RG properties of minimal supergravity again found this numerical coincidence (see, e.g., Refs. [88,85]). However, none of these studies interpreted these results as allowing natural multi-TeV scalars. This claim was first made in Ref. [17], where the issues relevant to the inclusion or exclusion of $c_{h_{t}}$ were carefully addressed, the naturalness bounds were investigated numerically using a full two-loop analysis, and the top mass required for a weak scale focus point was found to coincide (within experimental uncertainties) with the measured top mass. In this paper, the general requirements for focus point supersymmetry were also derived, and the potential for this behavior to solve and ameliorate the supersymmetric flavor and $C P$ problems was noted.

The peculiar value of the top quark mass thus highlights a question, which, for any other mass, would be of only academic interest: should the sensitivity to $h_{t}$ (and other standard model parameters) be included in calculations of fine tuning? Note that whether a parameter has been measured or not has no bearing on whether its sensitivity coefficient should be included. For example, if in the future the $\mu$ parameter is measured to be $10^{10} \mathrm{GeV}$ to arbitrarily high accuracy, but our theoretical understanding of electroweak symmetry breaking has not advanced, $c_{\mu}$ should still be included in measures of naturalness, and the weak scale should be considered (highly) fine tuned. (Not all naturalness studies take this view-see below.)

To address this question, we must first acknowledge the inescapable model dependence in any naturalness prescription. In any supersymmetry study, some fundamental framework must be adopted. In studies of other topics, however, there exists, at least in principle, the possibility of a modelindependent study, where no correlations among parameters are assumed. This model-independent study is the most general possible, in that all possible results from any other (model-dependent) study are a subset of the modelindependent study's results. In studies of naturalness, however, the correlations determine the results, and there is no possibility, even in principle, of a model-independent study in the sense described above. As an example, consider a study investigating models where the minimal supergravity assumptions, in particular, the assumption of scalar universality, are relaxed. In such models, the correlations required by the focus point mechanism are absent. This study therefore misses this possibility, and should conclude that it is never possible to raise all scalar masses far above the $\mathrm{TeV}$ level [although the scalar masses of the first and second generations may be as large as $\mathcal{O}(10 \mathrm{TeV})]$.
The model dependence of naturalness is present even in the most general statements concerning fine tuning. It is often assumed that, since the weak scale is $\sim 100 \mathrm{GeV}$, supersymmetry parameters of order $100 \mathrm{GeV}$ will yield a 1 part in 1 fine tuning in the electroweak scale. However, this is at odds with a low energy effective field theory perspective. From such a point of view, the Higgs boson mass receives radiative corrections $\Delta m_{h}^{2} \sim m_{\text {SUSY }}^{2} / 16 \pi^{2}$, so demanding a 1 part in 1 fine tuning would apparently allow supersymmetric masses of order $m_{\text {SusY }} \sim 1 \mathrm{TeV}$. The resolution is that the first statement implicitly assumes a fundamental theory at some high scale, such as $M_{\mathrm{GUT}}$, with fundamental parameters defined at this high scale. The radiative correction is then more precisely $\Delta m_{h}^{2} \sim m_{\mathrm{SUSY}}^{2} \ln \left(M_{\mathrm{GUT}} / M_{\text {Weak }}\right) / 16 \pi^{2}$, and the large logarithm offsets the loop factor suppression, yielding a 1 part in 1 fine tuning for $100 \mathrm{GeV}$ supersymmetry masses.

What about the top quark Yukawa? From a low energy point of view, one should include all the parameters of the Lagrangian, including $h_{t}$. However, by assuming some underlying high energy motivation by defining our parameters at $M_{\mathrm{GUT}}$, we have already abandoned a purely low energy perspective. Once we consider the high energy possibilities, the case is not so clear. For example, $h_{t}$ may be fixed to a specific value (or one of a set of discrete values) in a sector of the theory unrelated to supersymmetry breaking. An example of this is weakly coupled string theory, where $h_{t}$ may be determined by the correlator of three string vertex operators and would therefore be fixed to some discrete value determined by the compactification geometry. ${ }^{7}$ In such a scenario, it is clearly inappropriate to artificially vary $h_{t}$ continuously to determine the sensitivity of the weak scale to variations in $h_{t}$. This and other examples leading to the same conclusion were previously described in Ref. [18].

Clearly, no definitive answer can be given without improved knowledge of the fundamental theories of flavor and supersymmetry breaking. Without this knowledge, neither choice is beyond reproach. However, given the plausible suggestions from high energy frameworks that the standard model parameters may be fixed in ways unrelated to supersymmetry breaking, it is well worth considering the implications of relaxing the requirement that the weak scale be insensitive to variations in standard model couplings. Once we take this approach, we find it highly suggestive that the measured value of the top quark mass, along with the simplest of scalar mass boundary conditions, is exactly what is required to decouple scalars naturally and relieve the longstanding low energy problems of supersymmetry.

\section{Sensitivity vs fine tuning}

The approach of the early papers was criticized in a series of papers by Anderson and Castaño [80]. They pointed out

\footnotetext{
${ }^{7}$ Of course, one might argue that string theory may fix all parameters, including those that break supersymmetry. Taken to an extreme, then, no variables are free, and no definition of naturalness is possible. Such an approach is equivalent to the strongest possible anthropic principle, and no more constructive.
} 
that it is possible in certain cases that all possible choices of a fundamental parameter yield large sensitivities. They argued that in such cases, only relatively large sensitivities should be considered fine tuned, and drew a distinction between the sensitivity parameters $c_{i}$ defined above, and finetuning parameters, which they defined as $\gamma_{i} \equiv c_{i} / \bar{c}_{i}$, with $\bar{c}_{i}$ an average sensitivity. These $\gamma_{i}$ were then combined to form an overall fine-tuning parameter.

We agree in principle with these arguments. In addition to the virtues noted by Anderson and Castaño, the normalization step has the feature that the fine-tuning is then insensitive to whether the fundamental parameter is defined to be $m_{0}$ or $m_{0}^{2}$, for example. However, the averaging procedure may also mask important features. In their study, Anderson and Castaño propose two possible definitions of $\bar{c}_{i}$ and show that they yield roughly equivalent results. One of these definitions is $\bar{c}_{i}=\int_{a_{i}} c_{i}$, where, as indicated, the average is taken over a line in parameter space, varying $a_{i}$ while holding all other parameters fixed. Adopting this prescription, in minimal supergravity for a fixed $m_{0}$, say, $\gamma_{m_{0}}$ will be qualitatively the same for top Yukawa couplings both at the focus point value and far from it: in the latter case, the sensitivity coefficients $c_{m_{0}}$ will be much larger, but so will $\bar{c}_{m_{0}}$. We believe this hides a physical effect-it is clear that for the focus point top Yukawa coupling, the weak scale is much less sensitive to variations in $m_{0}$, and this fact should be reflected in any definition of naturalness.

To fix this, while preserving the principle virtue of the sensitivity vs fine-tuning distinction, one could define $\bar{c}_{i}$ $\equiv \int_{\left\{m_{0}, M_{1 / 2}, \ldots, h_{t}, \ldots\right\}} c_{i}$, where one averages over all of parameter space, including points with different $h_{t}$. This then introduces one overall normalization factor for each $c_{i}$, and we have checked that our results are not qualitatively altered by such a procedure. It is clear, however, that this modified Anderson-Castaño prescription requires a definition of averaging region, which introduces additional subjectivity and complications. Given that our results are not substantially changed, we do not include this refinement.

\section{E. Naturalness vs likelihood}

Finally, an alternative definition of sensitivity coefficient has been proposed in a series of papers $[81,82]$. In these studies, the definition of Eq. (12) is replaced by

$$
c_{i} \equiv\left|\frac{\Delta a_{i}}{m_{Z}^{2}} \frac{\partial m_{Z}^{2}}{\partial a_{i}}\right|,
$$

where $\Delta a_{i}$ is the experimentally allowed range of $a_{i}$. The intent of this alternative definition is to encode the idea that naturalness is our attempt to determine which values of parameters are most likely to be realized in nature.

To contrast this definition with the conventional definition, consider, for example, the hypothetical scenario described above, in which our theoretical understanding of supersymmetry has not improved, but the $\mu$ parameter is measured to be $10^{10} \mathrm{GeV}$ with very high accuracy. In the standard definition of sensitivity coefficient, Eq. (12), the model is fine-tuned. In our view, this is as it should be: such a large $\mu$ parameter signals a highly unnatural situation, and would strongly suggest a deficiency in our theoretical understanding. However, by the definition of Eq. (14), $\Delta \mu$ is very small, and so the electroweak scale is not fine tuned, even though it is smaller than $\mu$ by many orders of magnitude.

Naturalness is not simply a measure of our experimental knowledge of the parameters of nature. Rather it is a measure of how well a given theoretical framework explains the parameters realized in nature. It is perfectly possible for experimentally likely ranges of parameters to be unnatural-this is what the gauge hierarchy and cosmological constant problems are-and to think that this unnaturalness can be reduced by improved experimental measurements misses this essential point.

While this is perhaps the most fundamental difference between these papers and our approach, we conclude with some additional comments concerning the most recent study of Romanino and Strumia [82], as this specifically addresses the question of the naturalness of multi-TeV scalars.

Naturalness must be calculated in a well-defined framework. For example, if we assume minimal supergravity, the focus point works because the initial value of $m_{H_{u}}^{2}$ is $m_{0}^{2}$, and the RG contribution is roughly $-m_{0}^{2}$, so the weak scale value vanishes, independent of $m_{0}$. The authors of Ref. [82] ask, "is a cancellation between $\left[m_{0}\right]$ and the radiative contributions to it more 'natural' than a cancellation between different soft terms?' In our approach, the answer is yes, because in minimal supergravity, the first two are controlled by the same parameter, whereas different soft terms cancel only for certain choices of two or more parameters. Stated in another way, the assumptions of minimal supergravity guarantee this cancellation just as the assumptions of local quantum field theory guarantee that the electron's charge is canceled by the positron's, and it makes no more sense to think of the former cancellation as fine-tuned than the latter-it is part of the assumed framework. ${ }^{8}$ Of course, contrived frameworks should be considered less promising, but once the framework is adopted, one should not vary from its underlying assumptions.

The authors of Ref. [82] also work in the context of minimal supergravity. However, they consider the sensitivity of the focus point mechanism to "uncertainties associated with an unknown sparticle spectrum between $200 \mathrm{GeV}$ and 1 TeV.' In our approach, the weak scale threshold corrections are fixed by the input parameters, and there is no remaining freedom for ad hoc adjustments of the sparticle spectrum.

\footnotetext{
${ }^{8}$ To be clear, we note that in the case of minimal supergravity and the focus point, the cancellation is, of course, not perfect, in contrast with the case of local quantum field theory and particle/antiparticle charges. However, for top quark masses within the current experimental bounds, the cancellation is complete enough that the sensitivity to multi-TeV $m_{0}$ is below or of order the sensitivity to the other $\mathcal{O}(100 \mathrm{GeV})$ fundamental parameters, and far below what might naively be expected.
} 
All threshold corrections are therefore already included in our analysis of sensitivity coefficients and in our results.

Finally, Romanino and Strumia (and others [27]) concentrate their discussion on the case $\tan \beta \approx 10$, which may leave an impression that the focus point mechanism is operational only for that specific value of $\tan \beta$. Reference [82] also analyzed the effects of uncertainties in $h_{t}\left(M_{\mathrm{GUT}}\right)$ on the focus point scenario. We reiterate that the RG trajectories of $m_{H_{u}}^{2}$ focus at the weak scale for any value of $\tan \beta \geq 5$. This was demonstrated numerically in Ref. [18]; in the Appendix, we prove it analytically. Thus the naturalness of multi-TeV scalars is guaranteed for virtually all values of $\tan \beta$ allowed by present constraints on the light Higgs boson mass.

The $\tan \beta$ independence of the focus point is far from trivial. The top quark mass and $\tan \beta \gtrsim 5$ fix the top quark Yukawa coupling at the weak scale. However, as $\tan \beta$ increases from moderate to large values, $h_{b}$ becomes relevant and has two effects: first on the RG evolution of $h_{t}$, and, second, directly on the RG trajectories of the top squark masses and $m_{H_{u}}^{2}$. It is easy to see that these effects oppose each other. A non-negligible $h_{b}$ increases $h_{t}\left(M_{\mathrm{GUT}}\right)$ and the average value of $h_{t}$ through its RG evolution, which tends to drive $m_{H_{u}}^{2}$ more negative. On the other hand, larger $h_{t}$ and $h_{b}$ decrease the average top squark mass, which pushes $m_{H_{u}}^{2}$ less negative.

What is remarkable, however, is that at one loop, ignoring negligible hypercharge effects, these effects exactly compensate each other, so that the focus point remains at the weak scale. This is demonstrated in the Appendix, where we show that the focus point scale may be written in terms of $h_{t}\left(M_{\text {Weak }}\right)$ only, without reference to $h_{t}\left(M_{\mathrm{GUT}}\right)$ or the RG trajectory of $h_{b}$. Thus, the variation of $h_{t}\left(M_{\mathrm{GUT}}\right)$ is irrelevant to analyses of the focus point: the focus point mechanism is guaranteed for all $\tan \beta \geqslant 5$, even though the RG trajectories of $h_{t}, h_{b}$ and the third generation squark masses may vary widely as $\tan \beta$ varies in this range.

\section{CONCLUSIONS}

Focus point supersymmetry is motivated by the remarkable "coincidence" that the precisely measured top quark mass implies that multi-TeV scalars are natural, given certain simple high scale boundary conditions. In this paper, we have considered several phenomenological consequences of focus point supersymmetry. We find that the possibility of all scalar masses being naturally above $1 \mathrm{TeV}$ has a number of desirable features:

The difficulties of many GUT models in accommodating both gauge coupling unification and proton decay constraints are reduced.

Constraints from EDM measurements on unknown phases are less stringent by one to two orders of magnitude, and current constraints may be satisfied with $\mathcal{O}(0.1)$ phases.

The Higgs boson mass is predicted to be at or above 115 $\mathrm{GeV}$ in focus point scenarios, consistent with current constraints and the recent evidence for a $115 \mathrm{GeV}$ Higgs boson at LEP. Such large masses are typically difficult to obtain without fine tuning, but are achieved naturally in focus point supersymmetry through heavy top and bottom squarks, without the need to appeal to large $C P$ violating phases or heavy gauginos.

In addition, we analyzed the implications of focus point supersymmetry for two other important constraints on supersymmetric theories, the muon MDM and $B \rightarrow X_{s} \gamma$. In particular, for the muon MDM, while an observable deviation from the standard model is consistent with focus point supersymmetry with high $\tan \beta$, a near future measurement consistent with the standard model will exclude conventional regions of minimal supergravity parameter space, and will strongly prefer focus point scenarios.

Finally, we have concluded this study with an extended discussion of various naturalness prescriptions. We have identified and highlighted a number of key differences between our prescription and others in the literature. By far the most ambiguous and important issue, in our view, is the question of whether one should include sensitivities to standard model couplings in attempts to quantify the success of supersymmetry in solving the gauge hierarchy problem. We have identified several scenarios in which such sensitivities should not be included. Perhaps most suggestive, however, is the fact that by excluding the sensitivity to standard model parameters, the measured top quark mass implies that multi$\mathrm{TeV}$ scalars are natural for the simplest possible boundary condition of universal scalar masses. If this is more than a coincidence, the top quark mass is our hint that the low energy problems of supersymmetry are but a mirage, and the mass scale of all squarks and sleptons actually lies well above a $\mathrm{TeV}$.

\section{ACKNOWLEDGMENTS}

We are grateful to C. Kolda and T. Moroi for collaboration in the early stages of this work and to F. Wilczek for fruitful discussions. We also thank G. Anderson, P. Chankowski, A. Romanino, and A. Strumia for extensive correspondence and conversations. This work was supported in part by the U.S. Department of Energy under contracts DE-FG02-90ER40542 and DE-AC02-76CH03000 and in part by funds provided by the U.S. Department of Energy under cooperative research agreement DF-FC0294ER40818. J.L.F. was funded in part by a Taplin membership.

\section{APPENDIX: DEPENDENCE OF FOCUS POINT ON YUKAWA COUPLINGS}

In this Appendix, we show that in minimal supergravity, the focus point scale is determined only by the gauge couplings and the weak scale value of the top Yukawa coupling $h_{t}$. More precisely, we show that the renormalization scale at which the $m_{H_{u}}^{2}$ contours meet may be written only in terms of $h_{t}$ at the weak scale, with no reference to the rest of the top quark Yukawa RG trajectory (e.g., its value at $M_{\mathrm{GUT}}$ ) or to the bottom Yukawa coupling $h_{b}$. This demonstrates that if the focus point is at the weak scale for, say, $\tan \beta=5$, it remains there for all $\tan \beta>5$. In Ref. [18] this was shown analytically for $h_{b} \ll h_{t}$ (moderate $\tan \beta$ ) and $h_{b}=h_{t}$ (high 
$\tan \beta$ ), and also numerically for all $\tan \beta$. Here we demonstrate this analytically for all $\tan \beta$, neglecting the tau Yukawa coupling, but making no assumptions about the relative magnitudes of $h_{t}$ and $h_{b}$. An abbreviated version of this proof was presented in Ref. [19].

To analyze the focus point, it is convenient to define

$$
\begin{aligned}
t & \equiv \frac{1}{2 \pi} \ln \left(\frac{Q}{M_{\mathrm{GUT}}}\right), \\
\alpha_{i} & \equiv \frac{g_{i}^{2}}{4 \pi}, \\
Y_{i} & \equiv \frac{h_{i}^{2}}{4 \pi} \\
m_{i}^{2} & \left.\equiv m_{i}^{2}\right|_{\mathrm{p}}+\Delta_{i}^{2},
\end{aligned}
$$

where $Q$ is the renormalization scale, ${ }^{9} g_{i}$ and $h_{i}$ are gauge and Yukawa couplings, respectively, and $m_{i}^{2}$ are scalar masses. Following the notation of Refs. $[13,17,18]$, we separate the scalar mass into $\left.m_{i}^{2}\right|_{\mathrm{p}}$, a particular solution to the RG equations, and $\Delta_{i}^{2}$, the remaining homogeneous part.

We now keep only the top and bottom Yukawa couplings, and neglect the small hypercharge difference in the $Y_{t}$ and $Y_{b}$ RG equations. With these approximations, the one-loop $\mathrm{RG}$ equations for the couplings are

$$
\begin{aligned}
& \dot{\alpha}_{3}=-3 \alpha_{3}^{2}, \quad \dot{\alpha}_{2}=\alpha_{2}^{2}, \quad \dot{\alpha}_{1}=\frac{33}{5} \alpha_{1}^{2}, \\
& \dot{Y}_{t}=Y_{t}\left[6 Y_{t}+Y_{b}-r(\alpha)\right], \\
& \dot{Y}_{b}=Y_{b}\left[Y_{t}+6 Y_{b}-r(\alpha)\right],
\end{aligned}
$$

where $\equiv d / d t$ and $r(\alpha) \equiv \frac{16}{3} \alpha_{3}+3 \alpha_{2}+\frac{13}{15} \alpha_{1}$ is a function of gauge couplings only. The homogeneous scalar mass evolution is given by

$$
\dot{\boldsymbol{\Delta}}^{2}=\mathbf{N} \boldsymbol{\Delta}^{2}
$$

where

$$
\mathbf{N}=\left[\begin{array}{ccccc}
3 Y_{t} & 3 Y_{t} & 3 Y_{t} & 0 & 0 \\
2 Y_{t} & 2 Y_{t} & 2 Y_{t} & 0 & 0 \\
Y_{t} & Y_{t} & Y_{t}+Y_{b} & Y_{b} & Y_{b} \\
0 & 0 & 2 Y_{b} & 2 Y_{b} & 2 Y_{b} \\
0 & 0 & 3 Y_{b} & 3 Y_{b} & 3 Y_{b}
\end{array}\right]
$$

and $\Delta^{2}=\left[\Delta_{H_{u}}^{2}, \Delta_{U_{3}}^{2}, \Delta_{Q_{3}}^{2}, \Delta_{D_{3}}^{2}, \Delta_{H_{d}}^{2}\right]^{T}$, with $U_{3}, Q_{3}$, and $D_{3}$ the third generation squark multiplets, and $H_{u}$ and $H_{d}$ the up- and down-type Higgs multiplets.

${ }^{9}$ Notice that we have rescaled the variable $t$ relative to its conventional definition, in order to simplify the equations to follow.
Equation (A8) is a set of five coupled differential equations, but the simple form of $\mathbf{N}$ implies that the RG evolution of three degrees of freedom is trivial. To make this explicit, define

$$
\begin{gathered}
\frac{\Delta^{2}(t)}{m_{0}^{2}} \equiv c_{1}(t)\left[\begin{array}{l}
3 \\
2 \\
1 \\
0 \\
0
\end{array}\right]+c_{2}(t)\left[\begin{array}{l}
0 \\
0 \\
1 \\
2 \\
3
\end{array}\right]+c_{3}(t)\left[\begin{array}{r}
1 \\
-1 \\
0 \\
0 \\
0
\end{array}\right] \\
+c_{4}(t)\left[\begin{array}{r}
0 \\
1 \\
1 \\
1 \\
0
\end{array}\right]+c_{5}(t)\left[\begin{array}{r}
0 \\
0 \\
0 \\
-1 \\
1
\end{array}\right],
\end{gathered}
$$

where we have factored out an overall mass scale $m_{0}$ to make the $c_{i}$ dimensionless. Equation (A8) then reduces to

$$
\begin{aligned}
& \dot{c}_{1}=Y_{t}\left(6 c_{1}+c_{2}\right), \\
& \dot{c}_{2}=Y_{b}\left(c_{1}+6 c_{2}\right),
\end{aligned}
$$

and $\dot{c}_{3}=\dot{c}_{4}=\dot{c}_{5}=0$.

We now solve these equations in full generality. Equations (A11) form a linear homogeneous system of first-order ordinary differential equations with variable coefficients. No general method of solution exists for such systems [89]. However, in this case, the variable coefficients $Y_{t}$ and $Y_{b}$ satisfy Eqs. (A6) and (A7), and this allows us to integrate these equations after a well-chosen ansatz for the form of the $c_{i}$.

Let us make a change of variables [19]

$$
\begin{aligned}
& c_{1}(t)=c_{1}^{0}+Y_{t}(t) p(t), \\
& c_{2}(t)=c_{2}^{0}+Y_{b}(t) q(t),
\end{aligned}
$$

where $c_{i}^{0} \equiv c_{i}(0)$ and the boundary condition for the new variables $p$ and $q$ is $p(0)=q(0)=0$. Substituting these forms for $c_{1}$ and $c_{2}$ into Eqs. (A11) and using the Yukawa RG Eqs. (A6) and (A7), we find

$$
\begin{aligned}
& \dot{p}=Y_{b}(q-p)+r p+6 c_{1}^{0}+c_{2}^{0}, \\
& \dot{q}=Y_{t}(p-q)+r q+6 c_{2}^{0}+c_{1}^{0} .
\end{aligned}
$$

The difference of Eqs. (A14) and (A15) yields a simple first order linear inhomogeneous differential equation for $p-q$

$$
\frac{d}{d t}(p-q)=-\left(Y_{t}+Y_{b}-r\right)(p-q)+5\left(c_{1}^{0}-c_{2}^{0}\right)
$$

which integrates to [19] 


$$
p-q=5\left(c_{1}^{0}-c_{2}^{0}\right) e^{-\int\left(Y_{t}+Y_{b}-r\right)} \int e^{\int\left(Y_{t}+Y_{b}-r\right)} .
$$

To solve for $p$ or $q$, substitute Eq. (A17) into Eq. (A14) or Eq. (A15), respectively. The resulting differential equation is again easily solved, and the solution for $p$ is

$$
\begin{aligned}
p(t)= & \left(6 c_{1}^{0}+c_{2}^{0}\right) e^{\int r} \int e^{-\int r}-5\left(c_{1}^{0}-c_{2}^{0}\right) e^{\int r} \\
& \times \int\left[Y_{b} e^{-\int\left(Y_{t}+Y_{b}\right)} \int e^{\int\left(Y_{t}+Y_{b}-r\right)}\right] .
\end{aligned}
$$

The final solution for $c_{1}(t)$ is then [19]

$$
\begin{aligned}
c_{1}(t)= & c_{1}^{0}+Y_{t}(t) e^{\int_{0}^{t} d t_{1} r\left(t_{1}\right)} \\
& \times\left\{\left(6 c_{1}^{0}+c_{2}^{0}\right) \int_{0}^{t} d t_{1} e^{-\int_{0}^{t_{1}} d t_{2} r\left(t_{2}\right)}-5\left(c_{1}^{0}-c_{2}^{0}\right)\right. \\
& \times \int_{0}^{t} d t_{1} Y_{b}\left(t_{1}\right) e^{-\int_{0}^{t_{1}} d t_{2}\left[Y_{t}\left(t_{2}\right)+Y_{b}\left(t_{2}\right)\right]} \\
& \left.\times \int_{0}^{t_{1}} d t_{2} e^{\int_{0}^{t_{2}} d t_{3}\left[Y_{t}\left(t_{3}\right)+Y_{b}\left(t_{3}\right)-r\left(t_{3}\right)\right]}\right\}
\end{aligned}
$$

and $c_{2}(t)$ is obtained by interchanging $t \leftrightarrow b$, and $1 \leftrightarrow 2$.

The focus point scale $t_{F}$ is given by

$$
\Delta_{H_{u}}^{2}\left(t_{F}\right)=c_{3}+3 c_{1}\left(t_{F}\right)=0 \text {. }
$$

In the case of a universal scalar mass, the initial conditions are $c_{i}(0)=[3 / 7,3 / 7,-2 / 7,-1 / 7,-2 / 7]$. Equation (A19) then becomes

$$
c_{1}(t)=\frac{3}{7}+3 Y_{t}(t) e^{\int_{0}^{t} d t_{1} r\left(t_{1}\right)} \int_{0}^{t} d t_{1} e^{-\int_{0}^{t_{1}} d t_{2} r\left(t_{2}\right)} .
$$

Note the great simplification following from $c_{1}^{0}=c_{2}^{0}$. The focus point is therefore fixed by the constraint

$$
Y_{t}\left(t_{F}\right) e^{\int_{0}^{t_{F}} d t_{1} r\left(t_{1}\right)} \int_{0}^{t_{F}} d t_{1} e^{-\int_{0}^{t_{1}} d t_{2} r\left(t_{2}\right)}=-\frac{1}{9} .
$$

We see that $t_{F}$ depends on the entire RG trajectories of the gauge couplings and on $Y_{t}$ at the focus point, but is independent of the rest of the $Y_{t}$ trajectory and is also entirely independent of $Y_{b}$. For the physical top mass $m_{t}$ $\approx 174 \mathrm{GeV}$ and $\tan \beta \approx 5$, we know that the focus point is at the weak scale [17]. As we raise $\tan \beta, Y_{t}\left(t_{F}\right)=Y_{t}\left(t_{\text {Weak }}\right)$ remains approximately constant to reproduce the physical top quark mass, but $Y_{b}$ increases. Eventually, $Y_{b}$ will be large and the RG trajectory of $Y_{t}$ [and, of course, $Y_{t}\left(M_{\mathrm{GUT}}\right)$ ] will be modified accordingly. The scalar mass RG trajectories are then also modified. Remarkably, the analysis above shows that despite this, the focus point of the $m_{H_{u}}^{2}$ trajectories remains at the weak scale. The focus point therefore remains at the weak scale for all $\tan \beta \geq 5$, and, in particular, is independent of the GUT scale value of $Y_{t}$, as long as $Y_{t}$ at the weak scale remains fixed, as it must to be consistent with the measured top quark mass.

With the analytic solution at hand, it is now straightforward to generalize the focus point discussion to the case of scalar mass non-universality. Using the empirical relation Eq. (A22), we can write the focus point condition in the form

$$
3 c_{1}^{0}-c_{2}^{0}+3 c_{3}=0 .
$$

Any set of nonuniversal boundary conditions satisfying Eq. (A23) will exhibit a focus point at the weak scale, at least for a certain range of (moderate) values of $\tan \beta$. Furthermore, if in addition

$$
c_{1}^{0}-c_{2}^{0}=0
$$

then a weak scale focus point exists for any value of $\tan \beta$ $\geq 5$. The most general set of nonuniversal scalar boundary conditions satisfying both Eqs. (A23) and (A24) is [18]

$$
\left[\begin{array}{l}
m_{H_{u}}^{2} \\
m_{U_{3}}^{2} \\
m_{Q_{3}}^{2} \\
m_{D_{3}}^{2} \\
m_{H_{d}}^{2}
\end{array}\right]=m_{0}^{2}\left[\begin{array}{l}
1 \\
1+x \\
1-x \\
1+x-x^{\prime} \\
1+x^{\prime}
\end{array}\right]
$$

with both $x$ and $x^{\prime}$ arbitrary.
[1] L. Maiani, in Proceedings of the Gif-sur-Yvette Summer School, Paris, 1980, p. 3.

[2] E. Witten, Nucl. Phys. B188, 513 (1981).

[3] M. Veltman, Acta Phys. Pol. B B12, 437 (1981).

[4] R. K. Kaul, Phys. Lett. 109B, 19 (1982).

[5] H. Goldberg, Phys. Rev. Lett. 50, 1419 (1983).

[6] J. Ellis, J. S. Hagelin, D. V. Nanopoulos, and M. Srednicki, Phys. Lett. 127B, 233 (1983).

[7] S. Dimopoulos, S. Raby, and F. Wilczek, Phys. Rev. D 24, 1681 (1981).
[8] G. F. Giudice and R. Rattazzi, Phys. Rep. 322, 419 (1999)

[9] L. Randall and R. Sundrum, Nucl. Phys. B557, 79 (1999).

[10] A. Pomarol and R. Rattazzi, J. High Energy Phys. 05, 013 (1999).

[11] E. Katz, Y. Shadmi, and Y. Shirman, J. High Energy Phys. 08, 015 (1999).

[12] T. Moroi, Phys. Lett. B 447, 75 (1999).

[13] J. L. Feng and T. Moroi, Phys. Rev. D 61, 095004 (2000).

[14] S. Dimopoulos, G. F. Giudice, and A. Pomarol, Phys. Lett. B 389, 37 (1996). 
[15] T. Han and R. Hempfling, Phys. Lett. B 415, 161 (1997).

[16] T. Moroi and L. Randall, Nucl. Phys. B570, 455 (2000).

[17] J. L. Feng, K. T. Matchev, and T. Moroi, Phys. Rev. Lett. 84, $2322(2000)$.

[18] J. L. Feng, K. T. Matchev, and T. Moroi, Phys. Rev. D 61, 075005 (2000).

[19] J. L. Feng, K. T. Matchev, and T. Moroi, "Naturalness reexamined: Implications for supersymmetry searches," hep-ph/0003138.

[20] K. Agashe, Phys. Rev. D 61, 115006 (2000).

[21] J. L. Feng, N. Polonsky, and S. Thomas, Phys. Lett. B 370, 95 (1996).

[22] H. Georgi, H. R. Quinn, and S. Weinberg, Phys. Rev. Lett. 33, 451 (1974).

[23] J. Ellis, S. Kelley, and D. V. Nanopoulos, Phys. Lett. B 249, 441 (1990); J. Ellis, S. Kelley, and D. V. Nanopoulos, ibid. 260, 131 (1991); P. Langacker and M. Luo, Phys. Rev. D 44, 817 (1991); C. Giunti, C. W. Kim, and U. W. Lee, Mod. Phys. Lett. A 6, 1745 (1991); U. Amaldi, W. de Boer, and H. Furstenau, Phys. Lett. B 260, 447 (1991); F. Anselmo, L. Cifarelli, A. Peterman, and A. Zichichi, Nuovo Cimento A 104, 1817 (1991).

[24] H. Georgi and S. L. Glashow, Phys. Rev. Lett. 32, 438 (1974).

[25] J. L. Feng, K. T. Matchev, and F. Wilczek, Phys. Lett. B 482, 388 (2000).

[26] J. L. Feng, K. T. Matchev, and F. Wilczek, Phys. Rev. D 63, 045024 (2001).

[27] B. C. Allanach, J. P. Hetherington, M. A. Parker, and B. R. Webber, J. High Energy Phys. 08, 017 (2000).

[28] U. Chattopadhyay, A. Datta, A. Datta, A. Datta, and D. P. Roy, Phys. Lett. B 493, 127 (2000).

[29] S. Dimopoulos, S. Raby, and F. Wilczek, Phys. Lett. 112B, 133 (1982).

[30] V. Barger, M. S. Berger, and P. Ohmann, Phys. Rev. D 47, 1093 (1993); S. P. Martin and M. T. Vaughn, ibid. 50, 2282 (1994); I. Jack and D. R. Jones, Phys. Lett. B 333, 372 (1994); Y. Yamada, Phys. Rev. D 50, 3537 (1994); I. Jack, D. R. Jones, S. P. Martin, M. T. Vaughn, and Y. Yamada, ibid. 50, 5481 (1994).

[31] P. Langacker and N. Polonsky, Phys. Rev. D 47, 4028 (1993); 52, 3081 (1995); M. Carena, S. Pokorski, and C. E. Wagner, Nucl. Phys. B406, 59 (1993).

[32] J. Bagger, K. Matchev, and D. Pierce, Phys. Lett. B 348, 443 (1995).

[33] P. H. Chankowski, Z. Pluciennik, and S. Pokorski, Nucl. Phys. B439, 23 (1995); R. Barbieri, P. Ciafaloni, and A. Strumia, ibid. B442, 461 (1995).

[34] D. M. Pierce, J. A. Bagger, K. Matchev, and R. Zhang, Nucl. Phys. B491, 3 (1997).

[35] D. E. Groom et al., Eur. Phys. J. C 15, 1 (2000).

[36] S. Dimopoulos and H. Georgi, Nucl. Phys. B193, 150 (1981); N. Sakai, Z. Phys. C 11, 153 (1981).

[37] J. Hisano, H. Murayama, and T. Yanagida, Phys. Rev. Lett. 69, 1014 (1992); Nucl. Phys. B402, 46 (1993).

[38] K. Hagiwara and Y. Yamada, Phys. Rev. Lett. 70, 709 (1993).

[39] Y. Yamada, Z. Phys. C 60, 83 (1993).

[40] Y. Totsuka for the Superkamiokande Collaboration, talk presented at SUSY2K, CERN, Geneva, Switzerland, 2000.

[41] V. Lucas and S. Raby, Phys. Rev. D 55, 6986 (1997).
[42] T. Goto and T. Nihei, Phys. Rev. D 59, 115009 (1999).

[43] K. S. Babu and M. J. Strassler, "A reexamination of proton decay in supersymmetric grand unified theories," hep-ph/9808447.

[44] T. Goto and T. Nihei, "New constraint on the minimal SUSY GUT model from proton decay,', hep-ph/9909251.

[45] S. Dimopoulos and F. Wilczek, "Incomplete Multiplets In Supersymmetric Unified Models," Print-81-0600 (Santa Barbara); A. Masiero, D. V. Nanopoulos, K. Tamvakis, and T. Yanagida, Phys. Lett. 115B, 380 (1982); B. Grinstein, Nucl. Phys. B206, 387 (1982); J. Hisano, T. Moroi, K. Tobe, and T. Yanagida, Phys. Lett. B 342, 138 (1995); G. Altarelli, F. Feruglio, and I. Masina, "From minimal to realistic supersymmetric SU(5) grand unification," hep-ph/0007254.

[46] V. Lucas and S. Raby, Phys. Rev. D 54, 2261 (1996).

[47] S. M. Barr, Phys. Rev. D 59, 015004 (1999).

[48] L. J. Hall and S. Raby, Phys. Rev. D 51, 6524 (1995).

[49] S. M. Barr and S. Raby, Phys. Rev. Lett. 79, 4748 (1997).

[50] K. S. Babu and S. M. Barr, Phys. Rev. D 48, 5354 (1993); 50, 3529 (1994); 51, 2463 (1995).

[51] See, for example, R. Dermisek, A. Mafi, and S. Raby, Phys. Rev. D 63, 035001 (2001).

[52] T. Falk, K. A. Olive, M. Pospelov, and R. Roiban, Nucl. Phys. B560, 3 (1999).

[53] T. Moroi, Phys. Rev. D 53, 6565 (1996).

[54] E. D. Commins, S. B. Ross, D. DeMille, and B. C. Regan, Phys. Rev. A 50, 2960 (1994).

[55] P. G. Harris et al., Phys. Rev. Lett. 82, 904 (1999).

[56] CERN-Mainz-Daresbury Collaboration, J. Bailey et al., Nucl. Phys. B150, 1 (1979).

[57] R. M. Carey et al., Phys. Rev. Lett. 82, 1632 (1999).

[58] R. Carey, talk given at ICHEP00, Osaka, 2000.

[59] M. Grosse Perdekamp et al., Nucl. Phys. B (Proc. Suppl.) 76, 253 (1999).

[60] V. W. Hughes and T. Kinoshita, Rev. Mod. Phys. 71, S133 (1999).

[61] S. Bertolini, F. Borzumati, A. Masiero, and G. Ridolfi, Nucl. Phys. B353, 591 (1991).

[62] H. Anlauf, Nucl. Phys. B430, 245 (1994).

[63] J. A. Bagger, K. T. Matchev, and R. Zhang, Phys. Lett. B 412, 77 (1997).

[64] G. Degrassi, P. Gambino, and G. F. Giudice, J. High Energy Phys. 12, 009 (2000).

[65] K. Adel and Y. Yao, Phys. Rev. D 49, 4945 (1994); C. Greub and T. Hurth, ibid. 56, 2934 (1997); A. J. Buras, A. Kwiatkowski, and N. Pott, Phys. Lett. B 414, 157 (1997); Nucl. Phys. B517, 353 (1998).

[66] P. Ciafaloni, A. Romanino, and A. Strumia, Nucl. Phys. B524, 361 (1998); M. Ciuchini, G. Degrassi, P. Gambino, and G. F. Giudice, ibid. B527, 21 (1998); F. M. Borzumati and C. Greub, Phys. Rev. D 58, 074004 (1998); 59, 057501 (1999); "Lessons from $\bar{B} \rightarrow X_{s} \gamma$ in two Higgs doublet models," hep-ph/9810240.

[67] K. Chetyrkin, M. Misiak, and M. Munz, Phys. Lett. B 400, 206 (1997); 425, 414(E) (1998).

[68] A. Ali and C. Greub, Phys. Lett. B 361, 146 (1995); C. Greub, T. Hurth, and D. Wyler, ibid. 380, 385 (1996); Phys. Rev. D 54, 3350 (1996); N. Pott, ibid. 54, 938 (1996). 
[69] A. Strumia, Nucl. Phys. B532, 28 (1998); A. Czarnecki and W. J. Marciano, Phys. Rev. Lett. 81, 277 (1998).

[70] A. L. Kagan and M. Neubert, Eur. Phys. J. C 7, 5 (1999).

[71] CLEO Collaboration, S. Ahmed et al., ' $b \rightarrow s \gamma$ branching fraction and CP asymmetry,'” hep-ex/9908022.

[72] ALEPH Collaboration, R. Barate et al., Phys. Lett. B 429, 169 (1998).

[73] P. Igo-Kemenes, talk given at the LEPC open session, 2000; http://lephiggs.web.cern.ch/LEPHIGGS/talks

[74] ALEPH Collaboration, R. Barate et al., Phys. Lett. B 495, 1 (2000); see also talks by P. McNamara [ALEPH], P. Lutz [DELPHI], M. Felcini [L3], and A. Quadt [OPAL] at the LEPC meeting, 2000, http://committees.web.cern.ch/ Committees/LEPC/minutes/LEPC56.html

[75] J. Ellis, G. Ganis, D. V. Nanopoulos, and K. A. Olive, "What if the Higgs boson weighs 115-GeV?,', hep-ph/0009355.

[76] G. L. Kane, S. F. King, and L. Wang, "What will we learn if a Higgs boson is found?,' hep-ph/0010312.

[77] J. L. Feng, C. Kolda, and N. Polonsky, Nucl. Phys. B546, 3 (1999); J. Bagger, J. L. Feng, and N. Polonsky, ibid. B563, 3 (1999); J. A. Bagger, J. L. Feng, N. Polonsky, and R. Zhang, Phys. Lett. B 473, 264 (2000); Q. Shafi and Z. Tavartkiladze, ibid. 473, 272 (2000); J. Hisano, K. Kurosawa, and Y. Nomura, Nucl. Phys. B584, 3 (2000).
[78] J. Ellis, K. Enqvist, D. V. Nanopoulos, and F. Zwirner, Mod. Phys. Lett. A 1, 57 (1986).

[79] R. Barbieri and G. F. Giudice, Nucl. Phys. B306, 63 (1988).

[80] G. W. Anderson and D. J. Castaño, Phys. Lett. B 347, 300 (1995); Phys. Rev. D 52, 1693 (1995); 53, 2403 (1996).

[81] P. Ciafaloni and A. Strumia, Nucl. Phys. B494, 41 (1997); G. Bhattacharyya and A. Romanino, Phys. Rev. D 55, 7015 (1997); R. Barbieri and A. Strumia, Phys. Lett. B 433, 63 (1998); L. Giusti, A. Romanino, and A. Strumia, Nucl. Phys. B550, 3 (1999).

[82] A. Romanino and A. Strumia, Phys. Lett. B 487, 165 (2000).

[83] G. G. Ross and R. G. Roberts, Nucl. Phys. B377, 571 (1992).

[84] B. de Carlos and J. A. Casas, Phys. Lett. B 309, 320 (1993).

[85] P. H. Chankowski, J. Ellis, and S. Pokorski, Phys. Lett. B 423, 327 (1998); P. H. Chankowski, J. Ellis, M. Olechowski, and S. Pokorski, Nucl. Phys. B544, 39 (1999).

[86] L. Alvarez-Gaume, J. Polchinski, and M. B. Wise, Nucl. Phys. B221, 495 (1983).

[87] G. F. Giudice (private communication).

[88] M. Carena, M. Olechowski, S. Pokorski, and C. E. Wagner, Nucl. Phys. B419, 213 (1994); B426, 269 (1994).

[89] E. Kamke, Differetialgleichungen Lösungsmethoden und Lösungen (Chelsea Publishing Company, New York, 1959). 